\title{
Plattformtechnologie für die mobile, markerfreie Proteindetektion
}

\author{
Sabrina Jahns ${ }^{1}$, Lars Thorben Neustock ${ }^{1,2}$, Elmira Moussavi ${ }^{1}$, Martina Gerken ${ }^{1}$ \\ ${ }^{1}$ Institut der Elektrotechnik und Informationstechnik, Christian-Albrechts-Universität zu Kiel, \\ Kaiserstr. 2, $24143 \mathrm{Kiel}$ \\ ${ }^{2}$ Electrical Engineering, Stanford University, 350 Serra Mall, Stanford, CA 94305-9505
}

\section{Zusammenfassung}

Wir präsentieren eine Plattformtechnologie zur mobilen, markerfreien Detektion von mehreren Proteinen gleichzeitig. Ein photonischer Kristall, der mit Liganden lokal funktionalisiert ist, dient hier als Sensor. Über ein kompaktes, Kamera-basiertes Messsystem wird die Proteinanbindung an die Sensoroberfläche in ein Intensitätssignal umgewandelt, über dessen Amplitude die Proteinkonzentration bestimmt werden kann. Um die Detektionsgrenze dieser Technologie weiter zu verbessern, werden hier photonische Kristalle mit einer multiperiodischen und aperiodischen Gitterstruktur simulativ und experimentell untersucht. Dafür werden die Gesamtempfindlichkeit und die Resonanzgüte jeder Struktur bestimmt und mit der bisher verwendeten monoperiodischen Struktur verglichen. Es konnte festgestellt werde, dass die Resonanzgüte von mono- über multi- bis aperiodisch sich deutlich verbessert. Eine Steigerung der Gesamtempfindlichkeit konnte nicht festgestellt werden. Jedoch konnte anhand von Analysen der elektrischen Feldverteilung innerhalb der verschiedenen Strukturen beobachtet werden, dass die Modenausbreitungen in den aperiodischen Strukturen stark lokalisiert wird und die elektrische Feldstärke in diesen "Hot-Spots" deutlich über der mittleren Feldstärke, die eine flächige Funktionalisierung repräsentiert, liegt. Diese lokale Resonanzausbildung konnte zudem bereits in ersten experimentellen Untersuchungen bestätigt werden.

Keywords: Optische Biosensoren, photonische Kristalle, multiperiodische und aperiodische Nanostrukturen, markerfrei

\section{Einleitung}

Krankheiten wie akuter Herzinfarkt und Diabetes sollten möglichst schnell erkannt und behandelt werden, um den Krankheitsverlauf des betroffenen Patienten positiv beeinflussen zu können [1, 2]. Dafür sind kompakte, schnell arbeitende, dezentrale und einfach zu benutzende Sensoren von hohem Interesse für Point-of-Care Anwendungen. Diese Sensoren sollten in der Lage sein, spezifisch mehrere Proteine für eine eindeutige Diagnose nachzuweisen [3-5]. Im Gegensatz zu markerbasierten Messmethoden wie z.B. Detektion über Fluoreszenzsignale benötigen die markerfreien Methoden keine weiteren Präparationsschritte der Reagenzien [6]. Generell wird bei den markerfreien Methoden über einen Signalwandler, auf dessen Oberfläche Liganden zum Fangen der zu detektierenden Proteine immobilisiert sind, die Bindung der Proteine in ein messbares Signal umgewandelt. Dieses kann elektrochemisch, kolorimetrisch oder optisch durchgeführt werden [7-9]. Es wird hier eine optische Plattformtechnologie zur mobilen, markerfreien und parallelen De- tektion von mehreren Proteinen auf einem Chip präsentiert. Hierfür dient ein photonischer Kristall als Signalwandler, dessen Oberfläche lokal mit mehreren und spezifischen Liganden funktionalisiert wird, wie schematisch in Abb. 1 gezeigt [10].

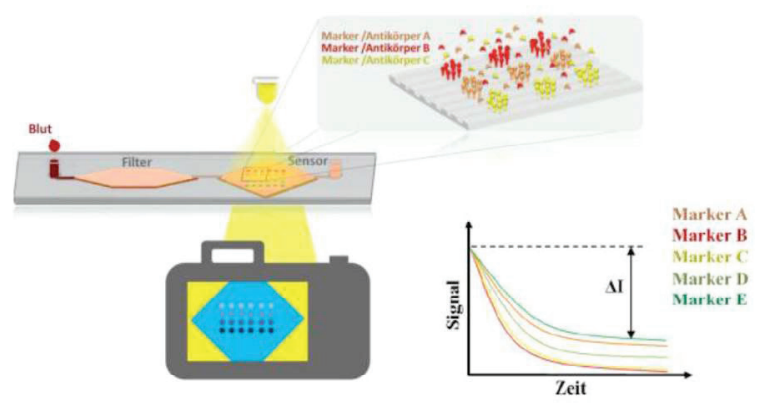

Abb. 1: Schema der Plattformtechnologie für die parallele, optische Konzentrationsmessung mehrerer Proteine in einer Blutprobe. 
Ein Tropfen Blut wird auf einen Wegwerfchip gegeben und das Plasma mit einem Filter getrennt. Das Sensorfeld enthält den funktionalisierten photonischen Kristall [11]. Photonische Kristalle sind Wellenleiter mit einer periodisch nanostrukturierten Oberfläche bestehend aus einem hochbrechenden, die-lektrischen Material. Dadurch können sich quasi-geführte Moden ausbilden, die sowohl im transmittierten als auch im reflektierten Licht-spektrum als Resonanzen sichtbar sind (Abb. 2). Aufgrund des evaneszenten Anteils der quasi-geführten Moden reagieren photonische Kristalle sensitiv auf Brechungsindexänderungen an der Sensoroberfläche mit einer Verschiebung der Resonanzposition.

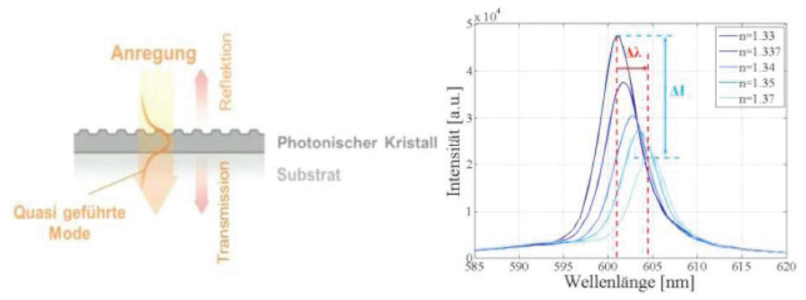

Abb. 2: Querschnitt der nanostrukturierten Oberfläche, die einen photonischen Kristall bildet, in dem quasi-geführte Moden auftreten (links). Resonanzverschiebung und beleuchtungsabhängige Intensitätsänderung hervorgerufen durch Brechungsindexänderung an der Oberfläche (rechts)

In einem Kamera-basierten Messsystem wird diese Resonanzverschiebung in eine Intensitätsänderung umgewandelt, durch welche wiederum die Proteinkonzentration bestimmt werden kann. Dazu wird die Systemantwort so entworfen, dass die Resonanz an einer abfallenden, spektralen Flanke liegt. Hier wird dieses durch die Verwendung einer farbigen LED als Anregungsquelle erreicht. Wie in Abb. 2 rechts zu sehen, resultiert die Brechungsindexerhöhung an der Oberfläche in einer starken Intensitätsänderung [12].

Für die optische Detektion werden die Liganden auf der $25 \times 25 \mathrm{~mm}^{2}$ großen Sensoroberfläche tropfenweise kovalent gebunden. Die Bindung des passenden Proteins erzeugt an den jeweiligen Positionen eine lokale Brechungsindexänderung und somit eine Intensitätsänderung des transmittierten Lichts, die dann mit der Kamera aufgenommen wird. Mit diesem System konnten wir die parallele und spezifische Anbindung der drei Proteine CD40 Ligand Antikörper (13.5 $\mu \mathrm{g} / \mathrm{ml})$, EGF Antikörper $(13.5 \mu \mathrm{g} / \mathrm{ml})$ und Streptavidin $(30 \mu \mathrm{g} / \mathrm{ml})$ anhand von 6 Ligandpositionen nachweisen (Abb. 2) [10].
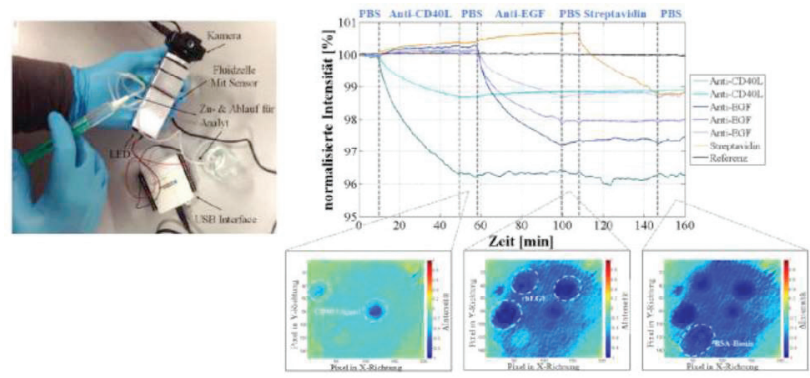

Abb. 3: Bild des Kamera-basierten Messsystems mit der Kamera, der LED, Optik innerhalb des metallischen Torsos, der Fluidzelle mit dem Sensor, Flügelkanülen für Zu- und Ablauf des Analyten und dem USB-Interface (links). Der Intensitätsverlauf und die entsprechenden Intensitätsbilder geben die Anbindungskurven bei einer Zugabe von zuerst CD40 Ligand Antikörper, dann EGF Antikörper und zum Schluss Streptavidin wieder. Es kommt zu keiner unspezifischen Bindung (rechts).

Es wird hier über zwei Verfahren zur Erhöhung der Empfindlichkeit berichtet, um das System für diagnostische Anwendungen, bei denen die relevante Proteinkonzentration häufig im $\mathrm{pg} / \mathrm{ml}$-Bereich liegt, attraktiver zu gestalten. Zuerst werden multiperiodische und aperiodische Nanostrukturen betrachtet und deren Gesamtempfindlichkeit und Resonanzgüte bestimmt [13, 14]. Als nächstes wird eine lokale Funktionalisierung der Nanostrukturen anhand von Analysen des elektrischen Feldes innerhalb dieser Strukturen simulativ erforscht.

\section{Spektrale Analyse von multiperiodischen und aperiodischen Nanostrukturen}

Hier werden multiperiodische und aperiodische Gitterstrukturen in photonischen Kristallen zur Steigerung der Empfindlichkeit untersucht. Multiperiodische Strukturen werden durch die logische ODER- Oberlagerung von zwei oder mehreren monoperiodischen Gittern erzeugt, wie aus Abb. 4 entnommen werden kann. 


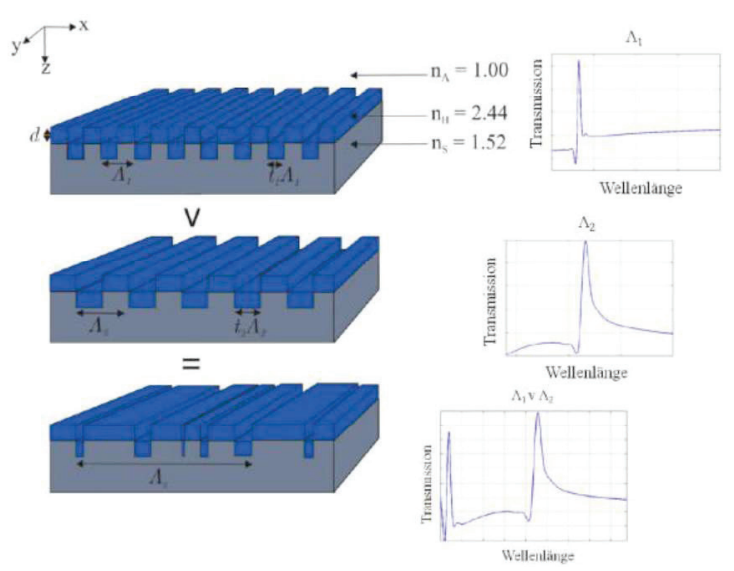

Abb. 4: Funktionsprinzip zur Erzeugung von multiperiodischen Gitterstrukturen (links) und die resultierenden Resonanzen zu den einzelnen Gitterstrukturen (rechts).

Multiperiodische Gitterstrukturen weisen mehrere Resonanzen auf, deren zentrale Wellenlänge durch die Ausgangsgitterstrukturen vorgegeben ist. Durch die Variation von verschiedenen Designparametern wie z.B. dem Tastverhältnis und der Anzahl der überlagerten Perioden wird die Struktur mit der höchsten Empfindlichkeit verglichen zu der Empfindlichkeit der bisher verwendeten monoperiodischen photonischen Kristalle identifiziert. Hierzu wurden die spektralen Eigenschaften wie der Güte $Q=\lambda_{R} / F W H M \quad\left(\lambda_{R}\right.$ : Resonanzwellenlänge; FWHM: Halbwertsbreite der Resonanz) und die Empfindlichkeit $S=\Delta \lambda / \Delta n$ $(\Delta \lambda$ : Wellenlängenverschiebung der Resonanz; $\Delta n$ : Brechungsindexänderung) auf Brechungsindexänderungen dieser Nanostrukturen simulativ und experimentell untersucht. Die aperiodischen Strukturen hingegen wurden nur simulativ untersucht. Dafür wurden exemplarisch binäre Sequenzen der drei mathematischen Folgen Rudin-Shapiro, Morse-Folge und Fibonacci in eindimensionale symmetrische Strukturen übertragen. Hierbei repräsentiert $A$ das Hochindexmaterial und $B$ das Substratmaterial (Tab. 1, Abb. 5).

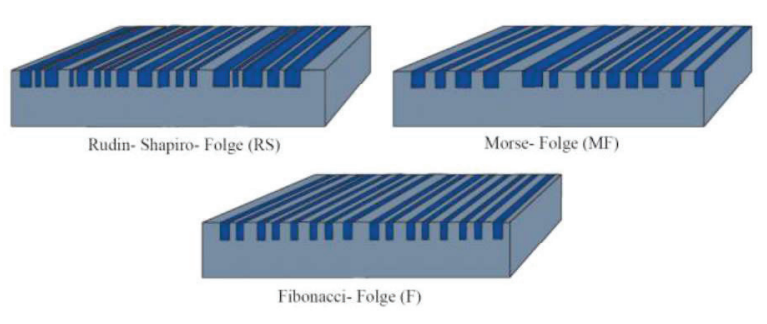

Abb. 5: Schemazeichnung der simulierten, aperiodischen Strukturen

Für die Simulationen wurden ein RCW (rigorous coupled wave) - Algorithmus in
Matlab implementiert und die in Tab. 1 aufgeführten Strukturen untersucht [15].

Tab. 1: Untersuchte multiperiodische und aperiodische Strukturen mit Periode $\Lambda$ und Tastverhältnis $t$

\begin{tabular}{|c|c|c|}
\hline & Typ & Beschreibung \\
\hline$M$ & $\begin{array}{l}\text { Mono- } \\
\text { periodisches } \\
\text { Gitter }\end{array}$ & $\Lambda=400 \mathrm{~nm} ; t=0,5$ \\
\hline M2 & $\begin{array}{l}\text { Multi- } \\
\text { periodisches } \\
\text { Gitter }\end{array}$ & $\begin{array}{l}\Lambda_{1}=350 \mathrm{~nm} ; \Lambda_{2}=450 \mathrm{~nm} ; \\
t_{1}=[0,14 ; 0,29 ; 0,42 ; 0,5 ; \\
0,57] ; \\
t_{2}=[0,14 ; 0,29 ; 0,42 ; 0,5 ; \\
0,57]\end{array}$ \\
\hline M3 & $\begin{array}{l}\text { Multi- } \\
\text { periodisches } \\
\text { Gitter }\end{array}$ & $\begin{array}{l}\Lambda_{1}=350 \mathrm{~nm} ; \Lambda_{2}=400 \mathrm{~nm} ; \\
\Lambda_{3}=450 \mathrm{~nm} ; \\
t_{1}=[0,14 ; 0,29 ; 0,42 ; 0,5 ; \\
0,57] ; \\
t_{2}=0,25 ; \\
t_{3}=[0,22 ; 0,33]\end{array}$ \\
\hline$R S$ & $\begin{array}{l}\text { Rudin- } \\
\text { Shapiro }\end{array}$ & $\begin{array}{l}\text { Substitution } A A \rightarrow A A A B, \\
A B \rightarrow A A B A, \quad B A \rightarrow B B A B, \\
B B \rightarrow B B B A ; \text { Bitlänge } \\
h=25 \mathrm{~nm} ; N=128 \\
\text { Serienkomponenten; } \\
\Lambda=6375 \mathrm{~nm} \text { Periode der } \\
\text { Superzelle }\end{array}$ \\
\hline$M F$ & Morse-Folge & $\begin{array}{l}\text { Substitution } A \rightarrow A B, \\
B \rightarrow B A ; B \text { itlänge } \\
h=50 \text { nm; } N=128 \\
\text { Serienkomponenten; } \\
\Lambda=12750 \mathrm{~nm} \text { Periode } \\
\text { der Superzelle }\end{array}$ \\
\hline$F$ & Fibonacci & $\begin{array}{l}\text { Substitution } A \rightarrow A B, \\
B \rightarrow A ; \text { Bitlänge } \\
h=50 \mathrm{~nm} ; N=144 \\
\text { Serienkomponenten; } \\
\Lambda=14350 \mathrm{~nm} \text { Periode } \\
\text { der Superzelle }\end{array}$ \\
\hline
\end{tabular}

Für die experimentellen Untersuchungen wurden die multiperiodischen Strukturen mit Hilfe von UV Nanoprägelithographie von einem Master auf Glassubstrate repliziert und $\mathrm{TiO}_{2}$ als Hochindexmaterial aufgedampft [10].

In Abbildung 6 sind exemplarisch die simulierten (links) und experimentell ermittelten (rechts) Spektren für das monoperiodische $M$ und ein multiperiodisches Gitter mit unterschiedlichen Analyten an der Oberfläche dargestellt. 

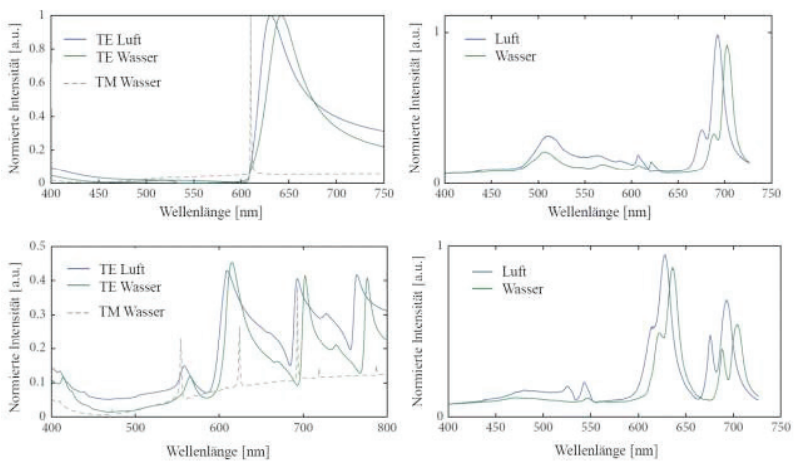

Abb. 6: Simulierte (links) und gemessene (rechts) Reflektionsspektren vom monoperiodischen M (oben) und multiperiodischen Gitter M3 (unten).

Aufgrund des geringeren Anteils des hochbrechenden Materials in den simulierten Strukturen unterscheiden sich die Resonanzpositionen in diesem Fall von den experimentellen. Jedoch sind die unterschiedlichen Resonanzen, die durch die überlagerten Ausgangsgitter hervorgerufen werden, sowohl in dem simulierten als auch in den gemessenen Reflektionsspektren sichtbar. Es konnte beobachtet werden, dass die Intensität der Resonanzen vom multiperiodischen im Vergleich zum monoperiodisch Ausgangsgitter deutlich schwächer ausfällt. Je mehr Perioden überlagert werden, desto schwächer werden die Intensitäten der dominanten Resonanzen. Über die Variation der Tastgrade jedes Ausgangsgitters, also dem Verhältnis von Stegbreite zu Periode, kann allerdings die Intensität jeder einzelnen Resonanz gesteuert und angepasst werden [14]. Desweiteren konnte keine Empfindlichkeitssteigerung im Vergleich zu dem monoperiodischen Gitter festgestellt werden. Jedoch nimmt die Güte der Resonanzen mit der Anzahl der überlagerten Perioden zu, was für eine bildgebende Sensorik von Vorteil ist.

Leider ergaben die aperiodischen Strukturen auch keine signifikante Empfindlichkeitssteigerung, jedoch wurde die Güte der Resonanzen noch einmal deutlich erhöht, wie aus den Tabellen 2 und 3 ersichtlich wird [15]. Allgemein steigt die Resonanzgüte mit der Unordnung in den Strukturen.

\section{Tab. 2: Empfindlichkeit S aller simulierten} (sim.) und gemessenen (exp.) Strukturen mit $\lambda_{R}$ der zentralen Wellenlänge für TE polarisiertes Licht

\begin{tabular}{|c|c|c|c|}
\hline Typ & $\begin{array}{c}\lambda_{R} \\
{[\mathrm{~nm}]}\end{array}$ & $\begin{array}{c}S \text { (sim.) } \\
{[\mathrm{nm} / \mathrm{RIU}]}\end{array}$ & $\begin{array}{c}S \text { (exp.) } \\
{[\mathrm{nm} / \mathrm{RIU}]}\end{array}$ \\
\hline$M$ & 700 & 33 & 32 \\
\hline$M 2$ & 600 & 15 & 22 \\
\hline
\end{tabular}

\begin{tabular}{|c|c|c|c|}
\hline & 770 & 38 & - \\
\hline \multirow{4}{*}{ M3 } & 550 & 12 & 13 \\
\cline { 2 - 4 } & 700 & 30 & 30 \\
\cline { 2 - 4 } & 800 & 41 & - \\
\hline \multirow{4}{*}{$R S$} & 450 & 16 & - \\
\cline { 2 - 4 } & 492 & 19 & - \\
\cline { 2 - 4 } & 533 & 25 & - \\
\cline { 2 - 4 } & 595 & 26 & - \\
\cline { 2 - 4 } & 740 & 49 & - \\
\hline \multirow{4}{*}{$M F$} & 418 & 19 & - \\
\cline { 2 - 4 } & 530 & 18 & - \\
\cline { 2 - 4 } & 544 & 20 & - \\
\cline { 2 - 4 } & 657 & 33 & - \\
\hline \multirow{2}{*}{$F$} & 515 & 23 & - \\
\cline { 2 - 4 } & 557 & 24 & - \\
\hline
\end{tabular}

Tab. 3: Güte $Q$ aller simulierten (sim.) und gemessenen (exp.) Strukturen mit $\lambda_{R}$ der zentralen Wellenlänge für TE polariziertes Licht

\begin{tabular}{|c|c|c|c|}
\hline Typ & $\lambda_{R}[\mathrm{~nm}]$ & $\mathrm{Q}$ (sim.) & $Q$ (exp.) \\
\hline$M$ & 700 & 19 & 35 \\
\hline \multirow{2}{*}{ M2 } & 600 & 16 & 32 \\
\cline { 2 - 4 } & 770 & 58 & - \\
\hline \multirow{4}{*}{ M3 } & 550 & 24 & 9 \\
\cline { 2 - 4 } & 700 & 68 & 26 \\
\cline { 2 - 4 } & 800 & 67 & - \\
\hline \multirow{4}{*}{ RS } & 450 & 62 & - \\
\cline { 2 - 4 } & 492 & 73 & - \\
\cline { 2 - 4 } & 533 & 95 & - \\
\cline { 2 - 4 } & 595 & 104 & - \\
\cline { 2 - 4 } & 740 & 150 & - \\
\hline
\end{tabular}

\section{Analysen des elektrischen Feldes innerhalb} der Nanostrukturen

Für die Proteindetektion mit nanostrukturierten Oberflächen ist die stochastische Verteilung der wenigen zu detektierenden Proteinen über die gesamte Sensorfläche von Nachteil. Durch die in diesem Abschnitt betrachtete lokale Funktionalisierung könnten die Proteine gezielt an die Positionen mit der höchsten elektrischen Feldstärke angebunden werden und somit das höchste Signal erzeugen. Durch die Betrachtung der elektrischen Feldverteilung in den multiperiodischen und aperiodischen Strukturen wird das Potential zur Empfindlichkeitssteigerung hier theoretisch untersucht. Über die elektrische Feldverteilung innerhalb und oberhalb einer Struktur kann die Ausbildung der einzelnen Moden untersucht und somit über die Berechnung der elektrischen Feldstärke $E$ an der Oberfläche auf die Empfindlichkeit der Struktur geschlossen werden $[16,17]$. Je höher das Betragsquadrat der elektrischen Feldstärke an der Oberfläche ist, desto empfindlicher reagiert die Struktur auf Brechungsindexänderungen. 

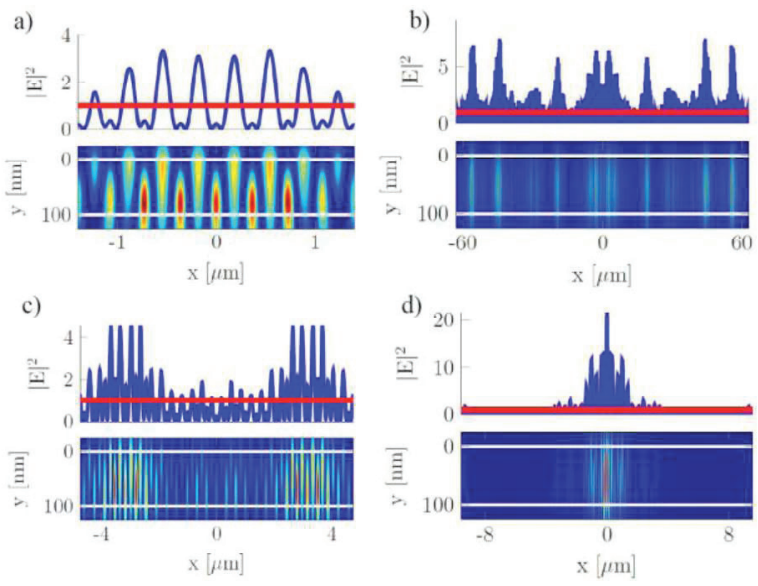

Abb. 7: Betragsquadrat der elektrischen Feldstärke an der Oberfläche und Betragsquadrat der elektrischen Feldverteilung innerhalb der Strukturen: a) zwei-periodisches Gitter, b) fünf-periodisches Gitter, c) RudinShapiro-Folge und d) Morse-Folge. Weiße Linien kennzeichnen die obere und untere Seite der Hochindexschicht.

Für Abbildung 7 wurde zunächst die elektrische Feldverteilung (unterer Teil) für jede Resonanz, die sich in den Strukturen ausbildet, mit Hilfe des RCW-Algorithmus betrachtet. Es sind sehr gut die evaneszenten Anteile der Moden sichtbar, die aus der Struktur herausragen und die photonischen Kristalle erst für die Sensorik nutzbar machen. Als nächstes wurde der Mittelwert des Betragsquadrates der elektrischen Feldstärke über die gesamte Strukturoberfläche berechnet (rote Linie). Dies entspricht der Systemantwort des photonischen Kristalls bei einer flächigen Funktionalisierung des Sensors. Anschließend wurde die elektrische Feldstärke ortsaufgelöst in x-Richtung berechnet (blaue Linie). Wie zu erkennen ist, ist die mittlere elektrische Feldstärke für alle Strukturen ziemlich ähnlich, was auch durch die Betrachtung der Empfindlichkeiten zu erwarten war. Jedoch sind die Moden in den verschiedenen Strukturen unterschiedlich stark lokalisiert und die maximalen Feldstärken teilweise deutlich höher als der Mittelwert, was eine deutliche Empfindlichkeitssteigerung in diesen Bereichen verspricht. Z.B. liefert die Resonanz bei $\lambda_{R}=600 \mathrm{~nm}$ der Morse- Folge die am stärksten lokalisierte Feldverteilung mit einer 20-fach höheren Feldstärke auf der Oberfläche als der Mittelwert (Abb. 7 d).
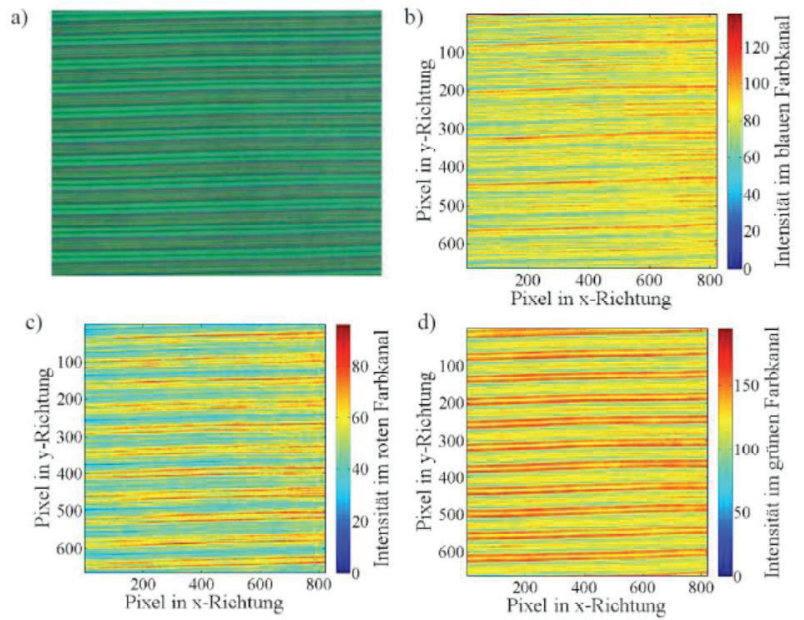

Abb. 8: a) Foto vom photonischen Kristall mit Morse-Folge; Falschfarbenbilder des b) blauen, c) roten und d) grünen Farbkanals.

Dieser Effekt wurde in ersten experimentellen Untersuchungen bestätigt, wie man in Abb. 8 sieht. Über Elektronenstrahllithographie wurde die Morse-Folge, wie oben beschrieben, in einen Nickel-Master geschrieben. Dann wurde die Struktur über das Prägeverfahren auf ein Glassubstrat übertragen und als Hochindexmaterial erneut $\mathrm{TiO}_{2}$ mit einer Schichtdicke von $85 \mathrm{~nm}$ aufgetragen. Für das Foto des so entstandenen photonischen Kristalls (Abb. 8 a) wurde der Kristall auf einen Mikroskoptisch zwischen zwei gekreuzte, lineare Polfilter platziert und mit der Mikroskoplampe bestrahlt. Dadurch wird nahezu das gesamte Anregungslicht unterdrückt und nur das Licht, welches mit den Moden in der Struktur interagiert und wieder auskoppelt, über ein Objektiv mit 40-facher Vergrößerung zum Kamerasensor geleitet [18]. Das Foto zeigt deutlich, dass sich mehrere Resonanzen verschiedener Wellenlänge ausbilden. Durch die Betrachtung der blauen, grünen und roten Farbwerte des Fotos wird dieser Eindruck bestätigt. Um eine Aussage über die Empfindlichkeit der realen Strukturen treffen zu können, müssen allerdings noch weitere Experimente durchgeführt werden.

\section{Schlussfolgerung}

Wir demonstrierten hier eine Plattformtechnologie, die eine markerfreie und spezifische Detektion von mehreren Proteinen gleichzeitig über ein kamera-basiertes Messsystem ermöglicht. Wir untersuchten die Gesamtempfindlichkeit und die Resonanzgüte von multiperiodischen und aperiodischen Nanostrukturen in photonischen Kristallen, um die Detektionsgrenze unseres Systems zu verbessern. Mit einer Erhöhung der Unordnung in der Gitterstruktur, d.h. von streng monoperiodisch über 
multiperiodisch zu aperiodisch, konnte eine Verbesserung der Resonanzgüte beobachtet werden. Eine Steigerung der Gesamtempfindlichkeit bei diesen Strukturen konnte generell nicht festgestellt werden. Jedoch ist die Ausbildung der Resonanzen in diesen Strukturen sehr stark lokalisiert, was mit einer lokalen Felderhöhung und somit mit einer potentiellen, lokalen Empfindlichkeitssteigerung einhergeht. Durch die gezielte Funktionalisierung dieser Bereiche mit den Liganden erscheint eine deutliche Verbesserung des Detektionslimits möglich.

\section{Danksagung}

Die Autoren bedanken sich beim European Research Council (ERC) für die finanzielle Unterstützung innerhalb des Projektes „PhotoSmart" (307800). Auch bedanken wir uns bei Karlsruhe Nano and Micro Facility (KNMF, http://www.kit.edu/knmf) für die Unterstützung bei der Fertigung der multiperiodischen und aperiodischen Gitterstrukturen.

\section{Referenzen}

[1] J. McCord, R. M. Nowak, P. A. McCullough, C. Foreback, S. Borzak, G. Tokarski, M. C. Tomlanovich, G. Jacobsen, W. D. Weaver, Circulation 104, 1483-1488 (2001); doi: $10.1161 / \mathrm{hc} 3801.096336$

[2] M. B. Schulze, K. Hoffmann, H. Boeing, J. Linseisen, S. Rohrmann, M. Möhlig, A. F. H. Pfeiffer, J. Spranger, C. Thamer, H.-U. Häring, A. Fritsche, H.-G. Joost, Diabetes Care 30, 510-515 (2007); doi: 10.2337/dc06-2089

[3] F. Wei, P. Patel, W. Liao, K. Chaudhry, L. Zhang, M. Arellano-Garcia, S. Hu, D. Elashoff, H. Zhou, S. Shukla, F. Shah, C.-M. Ho, D. T. Wong, Clinical Cancer Research 15, 4446-4452 (2009); doi: 10.1158/1078-0432.CCR-09-0050

[4] C.-S. Huang, V. Chaudhery, A. Pokhriyal, S. George, J. Polans, M. Lu, R. Tan, R. C. Zangar, B. T. Cunningham, Analytical Chemistry 84, 1126-1133 (2012); doi: 10.1021/ac202817q

[5] O. Bleher, A. Schindler, M.-X. Yin, A. B. Holmes, P. B. Luppa, G. Gauglitz, G. Proll, Analytical \& Bioanalytical Chemistry 406, 3305-3314 (2014); doi:10.1007/s00216-0137504-y

[6] P. Kozma, F. Kehl, E. Ehrentreich-Förster, C. Stamm, and F. F. Bier, Biosensors and Bioelectronics 58, 287-307 (2014); doi:10.1016/j.bios.2014.02.049

[7] Y. Wang, D. Li, W. Ren, Z. Liu, S. Dong, E. Wang, Chemical Communications 22, 25202522, (2008); doi: 10.1039/B801055B

[8] J. A. Lee, S. Hwang, J. Kwak, S. I. Park, S. S. Lee, K.-C. Lee, Sensors and Actuators B 129, 372-379, (2008); doi:10.1016/j.snb.2007.08.034

[9] M. A. Lifson, D. B. Roy, B. L. Millers, Analytical Chemistry 86, 1016-1022, (2014); doi: 10.1021/ac401523e

[10] S. Jahns, M. Bräu, B. Meyer, T. Karrock, S. B. Gutekunst, L. Blohm, C. Selhuber-Unkel, R.
Buhmann, Y. Nazirizadeh, M. Gerken, Biomedical Optics Express 6, 3724-3736 (2015); doi:10.1364/BOE.6.003724

[11] S. Jahns, S. B. Gutekunst, C. Selhuber-Unkel, Y. Nazirizadeh, M. Gerken, Microsystem Technologies, 1-6 (2015); doi: 10.1007/s00542-0152746-6

[12] S. Jahns, Y. Nazirizadeh, B. Meyer, S. B. . Gutekunst, C. Selhuber-Unkel, M. Gerken, IEEE SENSORS, 1-3 (2013); doi: 10.1109/ICSENS.2013.6688384

[13] L. Dal Negro, S.V. Boriskina, Laser \& Photonics Reviews 6, 178-218 (2012); doi: 10.1002/lpor.201000046

[14] C. Kluge, J. Adam, N. Barie, P. J. Jakobs, M. Guttmann, M. Gerken, Optics Express 22, A1363-A1371 (2014); doi: 10.1364/OE.22.0A1363

[15] L. T. Neustock, S. Jahns, J. Adam, M. Gerken, Journal of Sensors 501, 6174527 (2016); doi: $10.1155 / 2016 / 6174527$

[16] I. D. Block, P. C. Mathias, S. I. Jones, L. O. Vodkin, B. T. Cunningham, Applied Optics 48, 6567-6574 (2009); doi: 10.1364/AO.48.006567

[17] Y. Ding and R. Magnusson, "Resonant leakymode spectralband engineering and device applications," Optics Express 12, 5661-5674 (2004); doi: 10.1364/OPEX.12.005661

[18] Y. Nazirizadeh, U. Bog, S. Sekula, T. Mappes, U. Lemmer, M. Gerken, Optics Express 18, 19120-19128 (2010); doi: 10.1364/OE.18.019120 